\title{
Monastères entre Loire et Charente
}

\section{Yves Henigfeld}

\section{(2) OpenEdition}

\section{Journals}

Édition électronique

URL : http://journals.openedition.org/rao/3182

DOI : 10.4000/rao.3182

ISBN : 978-2-7535-5014-8

ISSN : 1775-3732

\section{Éditeur}

Presses universitaires de Rennes

Édition imprimée

Date de publication : 31 décembre 2015

ISBN : 978-2-7535-5012-4

ISSN : 0767-709X

\section{Référence électronique}

Yves Henigfeld, "Monastères entre Loire et Charente », Revue archéologique de l'Ouest [En ligne], 32 2015, mis en ligne le 28 avril 2016, consulté le 02 mars 2021. URL : http://journals.openedition.org/ rao/3182 ; DOI : https://doi.org/10.4000/rao.3182

@ Presses universitaires de Rennes 
du développement de cette spécialité en archéologie préventive depuis plusieurs années. Allant dans ce sens, il faut saluer la diversité des intervenants associant des spécialistes expérimentés à de nouveaux acteurs de la discipline comme des étudiants présentant leurs travaux. Cette diversité s'exprime également par la variété des organismes représentés au colloque (ministère de la Culture, Inrap, collectivités territoriales et entreprises privées) qui traduit le nouveau visage de l'archéologie en France. Ces actes du colloque de Lyon livrent ainsi un volume fondamental pour la recherche de petit mobilier, mobilier non céramique ou instrumentum comme on l'appelle pour les périodes protohistorique et antique. Le titre du colloque "Actualité de la recherche sur les mobiliers non céramiques de l'Antiquité et du haut Moyen Âge " a donc été conservé pour sa publication. Très ambitieux, il tient donc toutes ses promesses compte tenu des productions proposées. Néanmoins, on ne peut que regretter la faible représentation des articles propre au haut Moyen Âge, seulement trois sur 35, constat qui montre que les efforts à fournir pour réunir les spécialistes des différentes périodes sont encore vains.

Jean SOULAT (Laboratoire LandArc - Craham UMR 6273)

Treffort C., Brudy P. (dir.), 2013 - Monastères entre Loire et Charente, Rennes, PUR, 320 p. + cahier central de XXXII Pl. couleur h. t.

Piloté par Cécile Treffort et Pascale Brudy, avec la collaboration d'Anne Autissier, cet ouvrage de 320 pages est issu d'un colloque intitulé Archéologie monastique en pays charentais... qui s'est tenu à Saintes et Saint-Amantde-Boixe ( $1^{\text {er }}-3$ avril 2005) et de travaux réalisés dans le cadre d'un projet collectif de recherche (PCR) mené de 2001 à 2008 sur les Conditions d'implantation des monastères dans les pays charentais au Moyen Âge... Si le titre de l'ouvrage manifeste la volonté d'étendre le propos jusqu'à la Loire, force est de constater que les études de sites localisés en dehors des pays charentais (actuels départements de Charente et de Charente-Maritime) sont peu nombreuses (deux sites en Vendée, un en Maine-et-Loire...). L'essentiel de la publication porte en effet sur un espace géographique et culturel présenté comme cohérent réunissant le littoral atlantique et le fleuve Charente.

L'ouvrage est introduit par Cécile Treffort dans un chapitre (p. 7-9) restituant l'histoire de cette entreprise collective et interdisciplinaire ayant réuni une vingtaine de chercheurs d'origines institutionnelles diverses pendant près d'une décennie. Le programme de recherche a également donné lieu à une exposition itinérante intitulée $A \grave{A}$ la table des moines charentais, publiée en 2005. L'ambition de ces travaux était de réunir un ensemble de données disparates et parfois inédites dans un ouvrage alternant approches synthétiques et études monographiques. Ce dernier est organisé en cinq parties.

La première partie, intitulée "Histoires de moines" (p. 15-36), s'ouvre par une contribution de Cécile Treffort, qui dresse un bilan historiographique de la vie monastique en pays charentais $\mathrm{du} \mathrm{V}^{\mathrm{e}}$ au XII ${ }^{\mathrm{e}}$ siècle. Elle brosse un tableau contrasté de la documentation disponible et des principaux courants ayant ponctué l'histoire monastique régionale, depuis l'éclosion des établissements religieux suburbains à la fin de l'Antiquité jusqu'aux monastères créés à fin du XI et au XII ${ }^{\mathrm{e}}$ siècles avant l'installation, au XIII ${ }^{\mathrm{e}}$ siècle, des ordres mendiants en milieu urbain.

Dans une seconde contribution, consacrée au prieuré Sainte-Sonne de Chadenac en Charente-Maritime, Luc Bourgeois, Bernard Farago-Szekeres et Brigitte Véquaud confrontent la littérature épique médiévale et les actes de la pratique aux données archéologiques. La fouille a notamment montré que l'établissement religieux est installé à l'emplacement d'une nécropole mérovingienne et d'une église ou chapelle funéraire remontant au début du vi siècle. Outre les vestiges immobiliers, se sont aussi d'autres aspects de la vie matérielle, et particulièrement la céramique, qui sont ici abordés.

La deuxième partie de l'ouvrage, subdivisée en cinq chapitres, traite des "Logiques d'implantation » (p. 37-90) des communautés religieuses en pays charentais.

Les deux premiers correspondent à des approches territoriales. La contribution de Sylvie Dinet Refaldo porte sur les prieurés charentais des abbayes poitevines aux $\mathrm{x}^{\mathrm{e}}$ et $\mathrm{XI}^{\mathrm{e}}$ siècles, qui représentent, à eux seuls, près d'un dixième des installations répertoriées en pays charentais. Ce phénomène s'explique par une combinaison de facteurs géographique, politique et économique. Les abbayes poitevines qui s'implantent en Charente sont aussi les plus prestigieuses et correspondent à celles où la présence des ducs d'Aquitaine est la plus marquée. La plupart de ces prieurés sont logiquement installés au nord du fleuve de la Charente, au sud du diocèse de Poitiers. Ce sont les abords de Charroux et de Nanteuil-en-Vallée, les axes de la Charente et de ses 
affluents, les marais de l'Aunis et de Saintonge, ainsi que le chemin de Saint-Jacques-de-Compostelle qui constituent les principaux pôles d'attraction. La seconde approche territoriale correspond à un inventaire des implantations monastiques en marge des diocèses de Poitiers, Limoges, Angoulême, Bordeaux et Saintes. Dans cette contribution, Anne Autissier évoque les ordres religieux en présence, au rang desquels figurent notamment les Bénédictins, les ordres canoniaux, le mouvement érémitique du XII siècle, les Templiers et les Hospitaliers. Cet inventaire est suivi de trois contributions qui auraient peut-être pu être réunies en une seule, puisqu'elles sont consacrées au même site, et dont la présence au sein du chapitre consacré aux « Logiques d'implantation " n'est d'ailleurs pas pleinement justifiée. Il s'agit en l'occurrence du prieuré clunisien Saint-JeanBaptiste à Ronsenac (Charente) fondé au XI siècle, à une vingtaine de kilomètres au sud d'Angoulême. La première étude correspond à une approche historique et architecturale des lieux par Christian Gensbeitel. Elle est suivie d'une présentation, par Adrien Montigny, des interventions archéologiques qui se sont succédées pendant une trentaine d'années, puis d'une étude archéo-anthropologique proposée par Bernard Farago-Szekeres, consacrée à un " pourrissoir " installé dans la salle capitulaire, dans un espace de communication avec le cloître. Ce dispositif funéraire singulier, mais pas inédit, présente la particularité d'être constitué de deux coffres maçonnés jumelés pourvus de linteaux fixés à mi-hauteur, facilitant la décomposition des corps déposés successivement dans le courant du XIve et au début du $\mathrm{Xv}^{\mathrm{e}}$ siècle. On relèvera la présence, dans le fond de la tombe, d'un pichet funéraire orné de deux écussons.

La troisième section de l'ouvrage, intitulée "Les moines de la mer ", est consacrée, comme le suggère le titre, aux établissements installés sur le littoral atlantique. Elle est constituée de six études monographiques concernant quatre sites dont le traitement représente, à lui seul, près d'un tiers de l'ouvrage (p. 92-189).

La première contribution, que l'on doit à Jean-Paul Nibodeau et Laurent Prysmicki, porte sur l'abbaye des Châteliers sur l'île de Ré, sur la commune de la Flotteen-Ré (Charente-Maritime), dont les ruines marquent fortement le paysage insulaire. Il s'agit d'une fondation monastique cistercienne de la seconde moitié du XII ${ }^{\mathrm{e}}$ siècle. L'église, qui correspond au seul vestige encore en élévation à la Révolution, doit son salut à son pignon qui servait d'amer. Les dégagements amorcés à la fin du XIx ${ }^{e}$ siècle et les fouilles entreprises de 1968 à 1975 ont permis de reconstituer une grande partie du plan de l'église et des bâtiments claustraux. Le projet de mise en valeur et de restauration du site est à l'origine de plusieurs interventions archéologiques ponctuellement menées entre 1992 et 2004 qui ont permis de mieux documenter l'abbaye. L'église, le cloître, les bâtiments conventuels mais aussi et surtout les carreaux de pavement des sols galeries du cloître du XIII ${ }^{\mathrm{e}}$ au $\mathrm{xV}^{\mathrm{e}}$ siècle sont ici présentés de façon détaillée.

Le deuxième édifice classé parmi des sites " littoraux " (à juste titre? la question n'est pas vraiment discutée) correspond à l'abbaye de Maillezais aux $\mathrm{X}^{\mathrm{e}}$ et $\mathrm{xI}^{\mathrm{e}}$ siècles. Installé au cœur du Marais poitevin, au sud de l'actuel département de la Vendée, le site est implanté sur un îlot calcaire, à l'extrémité ouest du bourg et d'un éperon rocheux surplombant le "marais mouillé ", à une vingtaine de kilomètres de la côte. Jocelyn Martineau présente ici, avec la collaboration d'Emmanuel Barbier, les résultats d'une campagne de fouilles programmées qu'il a mené, de 2000 à 2002, aux abords de l'église, ainsi qu'une étude de bâti réalisée à l'occasion de travaux de restauration sur la partie romane de l'édifice. L'un des intérêts de la fouille a été de démontrer que l'abbaye a été édifiée sur un site occupé dès le $\mathrm{x}^{\mathrm{e}}$ siècle. Cette première occupation est attestée par la présence de vestiges de structures d'habitat et d'un front de taille de carrière, perçu en limite ouest du plateau, attribués à une période comprise entre la fin du $\mathrm{Ix}^{\mathrm{e}}$ et la fin du $\mathrm{x}^{\mathrm{e}}$ siècle. Cette datation repose sur des analyses ${ }^{14} \mathrm{C}$ et sur un mobilier céramique qui aurait mérité d'être sommairement présenté, au moins à titre de preuve. La contribution est par ailleurs largement consacrée à l'étude des vestiges en élévation de l'église et notamment de la nef et du massif occidental. L'analyse architecturale démontre l'existence d'un premier projet de construction, peut-être inachevé, immédiatement suivi par le chantier de construction des tribunes et du massif occidental. L'auteur émet l'hypothèse d'une première l'église datant du $\mathrm{x}^{\mathrm{e}}$ siècle, antérieure à l'abbatiale du début du XI ${ }^{e}$ siècle, interprétée comme l'église du castrum comtal qui aurait précédé l'abbaye, mentionné dans un récit rédigé vers 1070, par le moine Pierre de Maillezais.

Proposée par Éric Normand, la troisième monographie présentée dans ce chapitre est consacrée au site clunisien de Saint-Vivien en Charente-Maritime, localisé à une quinzaine de kilomètres au sud de La Rochelle, en position littorale, au contact d'une zone de marais. Elle présente les résultats d'une série de sondages et de plusieurs campagnes de fouille, essentiellement réalisées de 1998 à 2001, au sud de l'église paroissiale actuelle. Ces interventions ont révélé une modeste occupation préromane marquée par la présence de vestiges d'habitats et d'un fossé pouvant correspondre à la limite d'un premier espace funéraire. L'occupation monastique se divise en quatre grandes phases, marquées par la construction, au $\mathrm{XII}^{\mathrm{e}}$ siècle, d'un bâtiment transformé en logis au XIV siècle, avant une réorganisation du site à la fin du $\mathrm{XIV}^{\mathrm{e}}$ ou du début $\mathrm{du} \mathrm{Xv}^{\mathrm{e}}$ siècle. La datation s'appuie en grande partie sur l'étude du mobilier notamment céramique, qui, comme 
dans la contribution précédente, n’est pas présentée. Ces données archéologiques, conjuguées avec l'exploitation des sources textuelles, en particulier des compte rendus des visites de l'ordre, permettent à Éric Normand de restituer l'histoire du site. Vers 1135, Saint-Vivien dépendait du prieuré clunisien Saint-Martin de l'île d'Aix. Le premier bâtiment est interprété comme une possible grange, destinée à recueillir les produits de la paroisse et de ses alentours. Ce pôle économique devient ensuite un prieuré rural ou domus clunisienne, avant d'être transformé en un véritable prieuré conventuel, doté d'un cloître dans le courant du XIv ${ }^{e}$ siècle, avec l'installation, dans le contexte d'insécurité de la guerre de Cent Ans, d'une communauté entière provenant de la maison mère. Deux fragments de matrice de sceau retrouvés en fouille ont permis d'identifier deux prieurs du XIv ${ }^{\mathrm{e}}$ siècle de Saint-Martin de l'île d'Aix, ce qui montre qu'ils ont bien fréquenté le site. Ces objets sont étudiés, dans la contribution suivante, par Jean-Claude Bonnin, Éric Normand et Bruno Zélie, qui présentent par ailleurs une liste de 32 prieurs de Saint-Martin de l'île d'Aix, de la fin du XI ${ }^{\mathrm{e}}$ au début du XVII ${ }^{\mathrm{e}}$ siècle.

La dernière contribution évoque l'implantation en Saintonge, pendant la seconde moitié du $\mathrm{XI}^{\mathrm{e}}$ siècle, de bénédictins venus de l'abbaye de la Chaise-Dieu, par le biais de deux études consacrées au prieuré de SaintGemme en Charente-Maritime. Ces dernières auraient mérité d'être réunies dans une seule contribution de façon à éviter les répétitions et à proposer un plan plus cohérent. La première correspond, pour l'essentiel, à une étude architecturale, menée au début des années 2000, sur l'église et les bâtiments claustraux par Andreï Gh. Vlad. L'analyse des transformations architecturales de l'abbaye du troisième tiers $\mathrm{du} \mathrm{XI} \mathrm{I}^{\mathrm{e}}$ siècle jusqu'au $\mathrm{Xv}^{\mathrm{e}}$ siècle est accompagnée de restitutions graphiques. La seconde contribution est signée par Philippe Duprat. Il s'agit un essai de synthèse sur les multiples interventions archéologiques qui se sont succédées sur le site de Saint-Gemme. L'étude commence par une brève histoire du prieuré et se prolonge par un historique des « fouilles » qui se sont échelonnées entre 1926 et 1982, parfois de façon officieuse, comme cette intervention, en 1959, du garde-champêtre du village dans un enfeu du cloître. La présentation s'achève par l'évocation des résultats d'un diagnostic mené au printemps 2003 sur une galerie du cloître, ayant notamment révélé des niveaux préromans.

Après la mer, ce sont les installations en contexte fluvial qui font l'objet d'une quatrième partie, intitulée " Les moines du fleuve " (p. 191-247), comprenant quatre présentations.

L'abbaye Saint-Cybard d'Angoulême, installée dans un faubourg en contrebas de l'enceinte urbaine et en bordure de la Charente, est traitée dans une contribution dans laquelle Marie-Ève Scheffer présente les résultats d'une étude de bâti réalisée en 2002, en amont des travaux d'extension du Centre National de la Bande Dessinée. Cette étude montre qu'aux XIII ${ }^{e}-X{ }^{e}$ siècles, l'abbaye était organisée de façon bipolaire, autour d'un cloître et d'une cour à vocation "profane ». Ce sont notamment les vestiges du logis abbatial et de l'infirmerie du XIII ${ }^{e}$ siècle qui ont pu être étudiés, ainsi que d'autres bâtiments conventuels remaniés à plusieurs reprises. L'établissement a principalement souffert de sa transformation en papeterie au début du XIX ${ }^{\mathrm{e}}$ siècle. Notons que cette prestigieuse abbaye, dont l'origine est attribuée $\mathrm{au} \mathrm{VI}^{\mathrm{e}}$ siècle, était en grande partie méconnue au début des années 2000. L'auteure mentionne en note une série d'opérations archéologiques postérieures à la rédaction de l'article qui ont probablement amélioré la compréhension du site abbatial, avec, entre autres, la découverte, en 2008, de plusieurs sépultures alto-médiévales à l'emplacement du parvis occidental de l'église.

Les deux contributions suivantes concernent l'abbaye-auxDames de Saintes, dédicacée en 1047 par le comte d'Anjou Geoffroy Martel et son épouse Agnès de Bourgogne. Cet établissement, qui correspond à la seule abbaye féminine du diocèse, a joué un rôle de premier plan dans l'histoire de la Saintonge, tant par ses possessions que par les familles ou les lignages de ses nonnes et de ses abbesses. L'origine, le destin et l'importance de cet établissement installé à proximité de la ville, sur la rive droite de la Charente, sont évoqués par Alain Michaud. La connaissance de ce centre religieux a malheureusement souffert de la disparition de nombreuses archives. La réhabilitation et la transformation de l'abbaye en " cité musicale " sont à l'origine d'un programme de restauration ayant entraîné, entre 1986 et 1988, des fouilles archéologiques, dont les résultats sont présentés par Christian Vernou. Sa contribution concerne plus précisément les vestiges du haut Moyen Âge, constitués d'un bâtiment à abside attribué aux $\mathrm{V}^{\mathrm{e}}-\mathrm{VI}^{\mathrm{e}}$ siècles, découvert aux nord de l'abbatiale, et d'un ensemble de bâtiments associés à des sols, à un grand foyer central et à des structure en creux dans le carré claustral. L'interprétation de ces vestiges comme basilique dédiée à Saint-Martin et monastère paléochrétien à l'origine de l'abbaye du $\mathrm{XI}^{\mathrm{e}}$ siècle reste, pour l'heure, hypothétique.

Cette quatrième partie s'achève par la présentation, par Anaël Vignet, des fouilles archéologiques du cloître de l'abbaye de Saint-Amant-de-Boixe (2002-2005) situé entre le fleuve Charente et la forêt de Boixe, à $18 \mathrm{~km}$ au nord d'Angoulême. L'origine de cette abbaye, mentionnée pour la première fois en 888 , est attribuée à la présence du tombeau de l'ermite saint Amant au vi ${ }^{\mathrm{e}}$ siècle. L'église du monastère $\mathrm{du}$ début $\mathrm{du} \mathrm{XI}^{\mathrm{e}}$ siècle est reconstruite au $\mathrm{XII}^{\mathrm{e}}$ siècle. Les bâtiments sont rachetés en 1973 par la commune, avant d'être restaurés à partir de 1985, puis transformés, dans les 
années 2000, en centre d'interprétation dédié à l'architecture romane. C'est aussi dans ce cadre que le cloître a fait l'objet de plusieurs fouilles programmées de 2001 à 2005, dont les résultats forment l'essentiel de l'article. Les apports de cette fouille sont divers. Outre la mise en évidence de légères variations dans l'orientation des bâtiments, la fouille a également livré des sépultures attestant de modes d'inhumations diversifiés et de beaux ensembles mobiliers, partiellement illustrés dans l'article.

L'ouvrage s'achève sur une approche patrimoniale des bâtiments monastiques, dont le statut et la perception changent selon la période considérée, avec la désacralisation brutale des lieux à la Révolution, entrainnant de nouvelles affections jusqu’à leur réutilisation récente comme espace culturel. C'est là une ouverture originale qui est offerte au lecteur sous la forme de quatre contributions réunies dans une partie intitulée " Politiques patrimoniales" (p. 249-293).

La première correspond à un chapitre de synthèse consacré au patrimoine architectural monastique en PoitouCharentes. Véronique Dujardin y retrace « les processus et des étapes de protection au titre des monuments historiques " du $\mathrm{XIX}^{\mathrm{e}}$ siècle à nos jours. Les contributions suivantes concernent la mise en valeur de trois établissements différents que sont le centre d'interprétation de l'architecture romane de SaintAmant-de-Boixe (Charente) présenté par Marylise Ortiz et Anaël Vignet, l'abbaye de Fontevraud (Maine-et-Loire) par Daniel Prigent et l'abbaye Saint-Vincent-de-Nieul-surl'Autise (Vendée) par Richard Levesque.

L'ouvrage, auquel il manque peut-être une conclusion générale, s’achève sur une bibliographie régionale abondante (p. 295-307) et un appréciable index des noms de lieux (p. 309-313).

L'objectif de départ, qui était d'alterner synthèses et études de cas, n'est pas tout à fait atteint puisque l'ouvrage, ne comprend au final, que trois ou quatre approches territoriales sur la vingtaine de contributions recensées. Ce sont ici les études monographiques qui sont privilégiées et qui présentent souvent des données inédites, par exemple sur l'origine des établissements monastiques, notamment lorsqu'elles résultent d'opérations archéologiques récentes. On retiendra par ailleurs l'apport des études de bâti dans la connaissance de plusieurs bâtiments.

D'un point de vue formel, on peut regretter que les illustrations soient parfois difficilement lisibles, les cartes de synthèses et un certain nombre de plans étant présentés dans le corps du texte à une échelle trop réduite. Ce défaut est partiellement atténué par la présentation des mêmes documents, en couleur et à une échelle plus adaptée, dans les planches du cahier central.

Quoi qu'il en soit, on ne peut que saluer la parution de cet ouvrage qui couronne un travail collectif rondement mené et qui offre un bel état des connaissances sur un territoire donné. Puisse-t-il susciter de nouveaux travaux de recherche et être une source d'inspiration pour d'autres espaces régionaux.

Yves Henigfeld (Université de Nantes)

Burnouf Joëlle et Catteddu Isabelle, 2015 - Archéologie du Moyen Âge, Rennes, éditions Ouest-France et Inrap, 143 p. (ISBN 978-2-7373-6701-4).

L’ouvrage réalisé par Joëlle Burnouf et Isabelle Catteddu, publié aux éditions Ouest-France/Inrap s'adresse à un large public auquel les deux auteures veulent faire passer un message, le Moyen Âge ce n'est pas ce que vous croyez! et proposer la découverte d'un autre Moyen Âge en s'appuyant principalement sur les résultats des fouilles archéologiques de ces trente dernières années.

Pour ce faire, les auteures débutent leur ouvrage de manière originale en invitant le lecteur à s'interroger sur le temps qu'il faisait à l'époque et comment était l'environnement. Cette partie particulièrement didactique augure bien des renouveaux de la recherche climatique grâce aux apports des nombreuses fouilles archéologiques et des travaux interdisciplinaires qu' elles suscitent.
Ainsi, après avoir dressé rapidement à l'aide de quelques exemples un état des lieux en quatre étapes des changements climatiques et de leurs répercussions sur l'environnement et sur les sociétés, après avoir présenté les paysages déjà fortement aménagés depuis, au moins l'âge du Bronze et montré que l'interaction hommes-milieux fabrique une dynamique créatrice de changements, les auteures font pénétrer le lecteur dans les campagnes médiévales. Elles suivent alors une démarche chronologique pour, tour à tour, exposer l'agriculture et ses développements au cours du millénaire avec notamment l'introduction de nouvel outillage, le développement de l'élevage ou plus tard celui du maraîchage ou de la fruiticulture; les manières d'habiter avec ces différents schémas d'occupation des sols et sa diversité 